\title{
Wasser, Kräuter, Käse und eine Prise Rätoromanisch : Bemerkungen und Erkenntnisse zu Gessners Kontakten mit den Drei Bünden
}

\author{
Bernhard, Jan Andrea
}

DOI: https://doi.org/10.1515/9783110499056

Posted at the Zurich Open Repository and Archive, University of Zurich ZORA URL: https://doi.org/10.5167/uzh-195877

Book Section

Published Version

Originally published at:

Bernhard, Jan Andrea (2019). Wasser, Kräuter, Käse und eine Prise Rätoromanisch : Bemerkungen und Erkenntnisse zu Gessners Kontakten mit den Drei Bünden. In: Leu, Urs B; Opitz, Peter. Conrad Gessner (1516-1565) : Die Renaissance der Wissenschaften : The Renaissance of Learning. Berlin/Boston: De Gruyter Oldenburg, 335-352.

DOI: https://doi.org/10.1515/9783110499056 


\section{Wasser, Kräuter, Käse und eine Prise Rätoromanisch: Bemerkungen und Erkenntnisse zu Gessners Kontakten mit den Drei Bünden}

\section{Einleitung}

Im Rahmen des Forschungsprojektes „Das Buch in Graubünden. Herkunft, Gebrauch, Funktion, Sammlung und Wirkung von Büchern, Buchsammlungen und Bibliotheken in den Drei Bünden (1500-1800)“, ${ }^{1}$ das vom Institut für Kulturforschung Graubünden und der Kantonsbibliothek Graubünden gemeinsam verantwortet wird, bin ich in Maienfeld in der Bibliothek des Generalstabchefs Theophil Sprecher von Bernegg auf ein Exlibris von Conrad Gessner gestossen. „Sum Conradi Gesneri Tigurinj", ${ }^{2}$ schrieb er in das Werk Methodus seu ratio compendiaria perveniendi ad veram solidamque medicinam: mirifice ad Galeni libros recte intelligendos utilis (Basel 1541) des Tübinger Mediziners und Botanikers Leonhart Fuchs (1501-1566), mit dem Gessner in regelmässiger Korrespondenz stand (Abb. 1). ${ }^{3}$ Diese „Methodenlehre“, um zu einer „veram solidamque medicinam“ zu gelangen, führt mitten in Gessners Arbeit hinein: Fuchs wie Gessner hatten dieselbe Arbeitsmethode, nämlich die Pflanzen, die Tiere oder die Patienten selbst in Augenschein zu nehmen und zu untersuchen („Autopsie“), in gleichzeitiger kritischer Auseinandersetzung mit dem bisherigen Wissen. ${ }^{4}$ „Sich selbst einen Augenschein zu nehmen“, war schliesslich das Hauptmotiv, dass Gessner im Juni 1561 seine Reise in die Drei Bünde und deren Untertanenlanden antrat (Abb. 2).

Freilich liegt nahe, dass Gessner auf seiner Reise durch die Drei Bünde nicht nur da und dort „botanisierte“, sondern auch seine bereits bestehenden Kontakte pflegen und ausbauen konnte. Über Gessners Beziehungen zu Bünden ist bereits verschiedentlich publiziert worden, unter anderem über seine Freundschaft mit Johannes Fabricius Montanus (1527-1566) aus Bergheim (Elsass); ${ }^{5}$ auch über seine

\footnotetext{
1 Vgl. Bernhard (2013), S. 57-81.

2 Signatur: PBS HV2_18842 (Sprecher-Bibliothek Maienfeld). Ein weiterer Druck mit einem Exlibris Gessners liegt in der Kantonsbibliothek Graubünden, nämlich Xylanders M. Antonini imperatoris Romani et Philosophi de seipso seu vita sua libri XII (Zürich 1559) unter der Signatur: 04206.

3 Vgl. Leu (2016), S. 274.

4 Vgl. ibidem, S. $183 \mathrm{f} ., 328 \mathrm{ff}$.

5 Vgl. Fischer (1940), S. 322-336; Bonorand (1967), S. 482-488.
}

Jan-Andrea Bernhard, Unviersität Zürich, Zürich, Schweiz, e-mail: bernhard@theol.uzh.ch https://doi.org/10.1515/9783110499056-018 


\section{METHODVS SEV}

RATIO COMPENDIARIf perueniendi ad ueram folidam'ǵg :nedicin mirificè ad Galeni libros rectè intelligend. utilis, nuncrecens in lucem $x$ dita, L E O NA ARTO FVCHSI O, fcholx mediç Tubingenfis profeffore publico, autore.

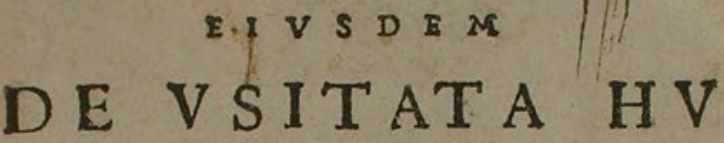

IVS TEMPORIS COMPO= nendorum mifcendorum'g medicamentorum ratione libri i I I ijs qui medicinam faciunt, uel, ut hodieloquuntur, praxim exercent, maximènecefiarij.

Indice duplici,latino \& græco, utroø copiofifsimo, adiecto.

\section{Cum inclytx Cxfarex Maieft, priuilegio, in annos quing.}

BASILEAE apud Michaëlem Ifingrinium, $M, D, X\llcorner\Sigma$.

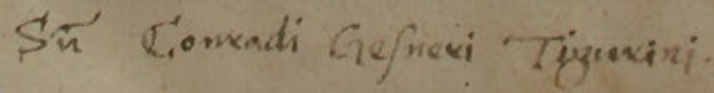

Abb. 1: Leonhart Fuchs: Methodus seu ratio compendiaria perveniendi ad veram solidamque medicinam: mirifice ad Galeni libros recte intelligendos utilis, Basel: Michael Isengrin, 1541 (Sprecher-Bibliothek, Maienfeld, Signatur: PBS HV2_18842). 


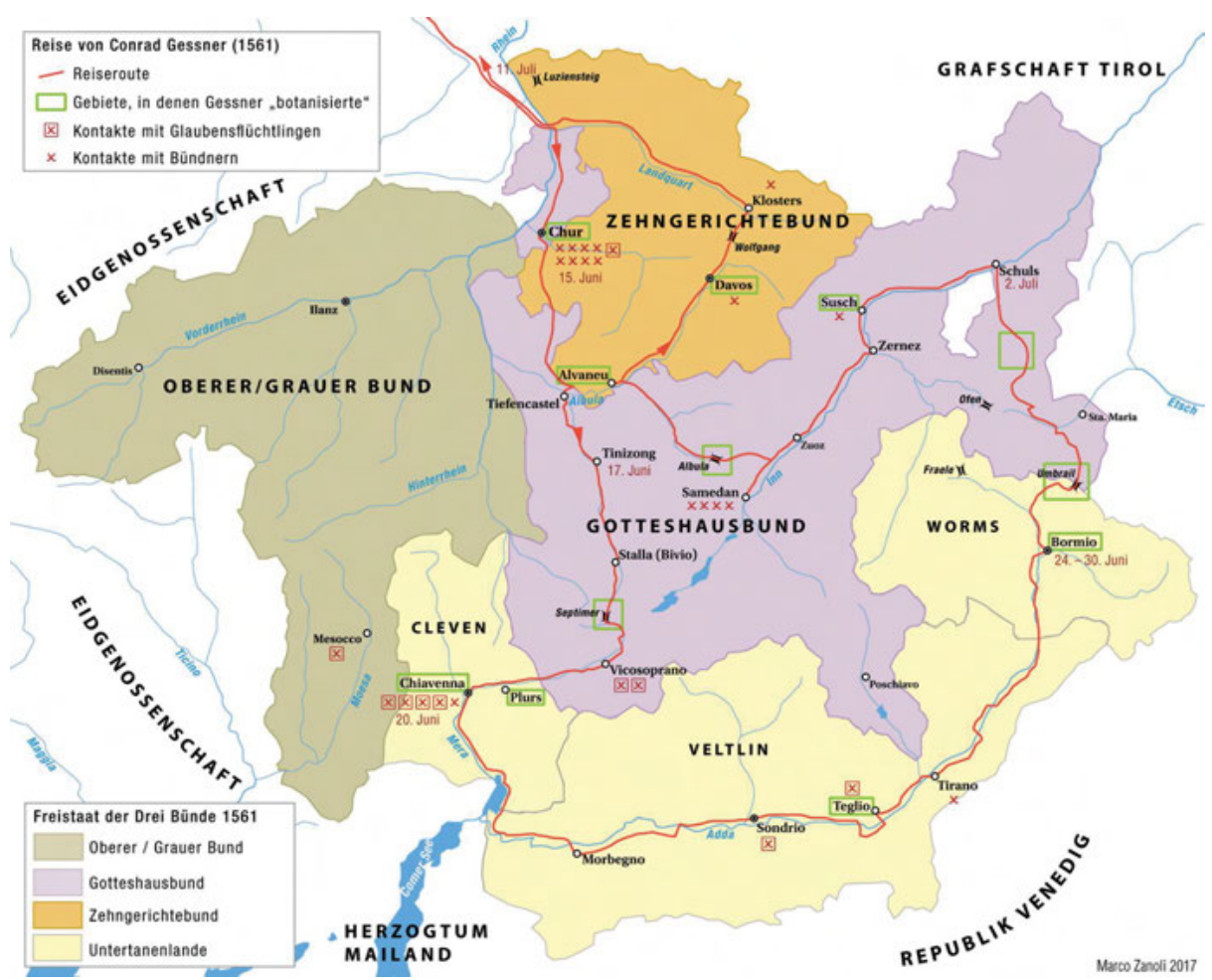

Abb. 2: Reiseroute Gessners durch die Drei Bünde und deren Untertanenlande im Juni/Juli 1561; eingezeichnet sind auch die Kontakte, die Gessner mit Bündnern hatte (C Marco Zanoli 2017).

„Bündner-Reise“ sind Studien erschienen, ${ }^{6}$ und schliesslich haben Forschungen zu seinem Album amicorum verschiedene bislang kaum bekannte Kontakte zu Bündner Gelehrten offenbart. ${ }^{7}$ Eine Gesamtschau der Kontakte Gessners mit Bünden ist dennoch ein Desideratum. Ein erster Versuch hat zwar Conradin Bonorand als Beilage zur Studie Die Engadiner Reformatoren: Philipp Gallicius - Jachiam Tütschett Bifrun - Durich Chiampell. Vortaussetzungen und Möglichkeiten ihres Wirkens aus der Perspektive der Reformation im allgemeinen (Chur 1987) geleistet, ${ }^{8}$ doch sind seither nicht nur zahlreiche neue Quellen entdeckt worden, sondern ist die Gessner-

\footnotetext{
6 Vgl. Döpp (2012), S. 16-38; Steiger (1978), S. 214-223; Brügger (1863), S. 34-43. Entgegen Brügger konnte Steiger die ursprüngliche Reiseroute nachweisen - wie Rosius à Porta in seiner Historia Reformationis Ecclesiarum Raeticarum (Chur/Lindau 1771) belegt, war im 18. Jahrhundert noch bekannt, dass Gessner von Bormio ins Unterengadin kam: „Hi Burmienses aquas exploraverunt, deinde in inferiorem Oengadinam deportati, ...“ (à Porta [1771], S. 336).

7 Vgl. Durling (1965), S. 134-159; Bonorand (1967), S. 483 ff.

8 Vgl. Bonorand (1987), S, 72-79. Bonorand hält 1987 fest, dass „eine umfassende Monographie des Universalgelehrten Konrad Gessner" noch ausstehe (S. 78).
} 
Forschung in den letzten 30 Jahren auch nicht untätig gewesen, so dass viele neue Aspekte zum Leben und Werk des Polyhistors bekannt geworden sind. Eine diesbezüglich eindrückliche Monographie zu Gessner ist sicher Urs B. Leus neue Biographie, erschienen aus Anlass von Gessners 500. Geburtstag.

Ein weiterer Aspekt rechtfertigt es, Gessners Kontakte mit den Drei Bünden genauer zu untersuchen: Es handelt sich bei den Drei Bünden nämlich um ein staatspolitisches, konfessionelles und geistesgeschichtliches Unikum im Europa des 16. Jahrhunderts. Die Drei Bünde haben sich den „Luxus“ geleistet, keinen Fürsten $\mathrm{zu}$ haben, und bereits Mitte der 20er Jahre des 16. Jahrhunderts hat der Bundstag, d. h. die Versammlung aller Gesandten der 52 Gerichtsgemeinden, die Verlagerung der Herrschaftsrechte vom Bischof von Chur auf die Gemeinden beschlossen. Dies bildete schliesslich die Grundlage dafür, dass in den Drei Bünden wie auch in ihren Untertanenlanden (Veltlin, Grafschaften Chiavenna und Bormio) ein bikonfessionelles Staatsgebiet entstanden ist, weil jede Gemeinde selbst entscheiden konnte, ob sie sich der Reformation anschliessen oder beim alten Glauben bleiben wolle; zudem gab es einen Minderheitenschutz für alle Personen beiderlei Geschlechts, sofern sie sich für die „päpstliche oder evangelische Religion“ entschieden hatten. ${ }^{9}$

Aus geistesgeschichtlicher Sicht sind die Drei Bünde gleichermassen in einer einzigartigen Situation gewesen: Über keine universitäre Einrichtung verfügend, war die Studentenperegrination eine grundlegende Form der Wissensaneignung, sowohl nach Norden als auch nach Süden, wie die Immatrikulationsverzeichnisse an zahlreichen Universitäten belegen. ${ }^{10}$ Damit ist die Bildungssituation in den Drei Bünde gewissermassen derjenigen im Fürstentum Siebenbürgen vergleichbar. ${ }^{11}$ Dort wie in den Drei Bünden fällt es gleichermassen auf, dass die Bildung des gemeinen Volkes erstaunlich gut war. So berichtet zum Beispiel Fabricius aus Chur an Bullinger darüber, dass die Prättigauer „bärglütt“ gute Bibelkenntnisse hätten. ${ }^{12}$

Solche „bärglütt“ lernte Gessner auf seiner Reise mehrere kennen. Er selber berichtet darüber an John Caius, nur einen Monat nach seiner Reise: „Abfui nuper toto fere mense in Alpibus Rhaeticis, quae Heluetiam Italiamque diuidunt: ad finitima Insubrum \& Venetorum loca per multos altissimosque montes profectus: itinere hinc (Tiguro) fere octidui: partim vt fontibus qui illic diuersi ac percelebres sunt fruerer, valetudinis meae causa: obiterque fontium naturas addiscerem: partim vt stirpes inquiererem, amicos inuiserem veteres, pararem nouos, animum a studijs relaxa-

9 Vgl. Bernhard (2016), S. 294-300; MacCulloch (2012), S. 74-84; Bundi (2003), S. 21-35; Head (2001), S. 88-97.

10 Es ist hier nur ganz summarisch vor allem auf die zahlreichen Studien von Felici Maissen und Conradin Bonorand zu verweisen; leider ist eine Datenbankerfassung der Studien aller Bündner an ausländischen Bildungseinrichtung bis heute ein Desideratum der Forschung.

11 Vgl. Tonk (1996), S. 113-125.

12 Vgl. Johannes Fabricius an Heinrich Bullinger, 21. Juni 1563, in: Schiess (1904-1906), Bd. 2, Nr. 521. 
rem, corpus exercitio firmarem, quae omnia mediocriter mihi (Dei gratia) contigerunt." 13

Ich bin mir bewusst, dass es im Rahmen dieses Aufsatzes nicht möglich ist, Gessners Kontakte mit den Drei Bünden in der notwendigen Tiefe und Breite vorzustellen. Um so mehr sind folgende Fragestellungen leitend: Was waren die Hauptmotive seines Interessens und seiner Kontakte? Inwiefern wird das bisherige Wissen ergänzt bzw. bestätigt? Welche Wirkung hatte Gessner auf Bünden?

\section{Gessners Kontakte mit den Drei Bünden}

Eine Zusammenstellung der bislang gesicherten oder mit grosser Wahrscheinlichkeit vermuteten Kontakte wurde bislang nie geleistet. Von den meisten Kontakten bzw. Begegnungen Gessners mit Bündnern - dazu werden auch italienischen Glaubensflüchtlinge gezählt, die infolge der Wiedereinführung der römischen Inquisition nach 1542 in die bündnerischen Untertanenlande und die Drei Bünde kamen, sich aber teilweise nur vorübergehend niederliessen ${ }^{14}$ - haben wir aus folgenden Quellen Kenntnis: Drucke, Korrespondenz, Buchwidmungen und Stammbücher. Natürlich wäre es, um ein umfassendes Bild von Gessners Kontakten zu haben, auch notwendig, in sämtlichen Briefen, Notizen und weiteren Drucken Gessners nach Namen aus dem Hoheitsgebiet der Drei Bünde zu suchen. Dies kann aber im Rahmen dieser Studie nicht geleistet werden. Immerhin wurde der Zettelkatalog von Rudolf Steiger (Promptuarium), der in der Abteilung Alte Drucke und Rara der Zentralbibliothek Zürich steht, minutiös ausgewertet.

In den heute greifbaren Quellen sind die Kontakte Gessners mit folgenden Personen (unter Angabe der betreffenden Jahre) greifbar: Johannes Pontisella (15371565), Albert Belfort (1541- 1545), Francesco Negri (1542- 1546), Agostino Mainardo (1543-1554), Baldassare Altieri (1547-1550), Pier Paolo Vergerio (1549-1562), Giovanni Beccaria (1550-1561), Celso Martinengo (1551), Johannes Fabricius (15531565), Giulielmo Gratarolo (1553-1565), Philipp Gallicius (1555- 1563), Iachiam Bifrun (1555- 1563), Friedrich v. Salis (1557-1563), Jacob Schucan (1557-1565), Johannes v. Juvalta (1557/58-1565), Scipione Calandrini (1558-1561), Zacharias Beeli (15591563), Hieronymus Brixius (1559-1563), Francesco Porto (1559-1565), Ludovico Quadrio (1561/62), Durich Chiampell (1561- 1563), Nicolò Guicciardi (1561-1564), Johannes Tscharner (1561-1564), Tobias Egli (1561- 1564/65), Johannes Möhr (15611564/65), Daniel Gugelberg v. Moos (1562), Nicolò Pestalozzi (1562-1564/65) und Stephan Heim (1564/65). ${ }^{15}$

13 Conrad Gessner an John Caius, 29. August 1561, in: Gessner (1577), f. 133v-134r.

14 Vgl. Bonorand (2000); Cantimori (1949).

15 Nicht erwähnt werden in diesem Zusammenhang die Kontakte Gessners mit den französischen Gesandten in Chur, wie z. B. mit Jean de Fraisse (1553-1554) oder Jean Jacques de Cambray (15581560); vgl. Schiess (1904-1906), Bd. 1, Nr. 266; Schiess (1904-1906), Bd. 2, Nr. 253. 


\section{Wissenstransfer}

Der Wissenstransfer zwischen Gessner und den genannten Personen war durch drei grundlegende Voraussetzungen bedingt: Briefkorrespondenz, Austausch von Büchern und der „Gelehrtenperegrination“. Die Untersuchung der Korrespondenz von Conrad Gessner offenbart, dass er mindestens mit zehn, wohl aber mehr Personen aus dem Hohheitsgebiet der Drei Bünde in Briefkontakt stand. ${ }^{16}$ So wissen wir beispielsweise, dass wohl auch Johannes Blasius aus Chur mit Gessner Briefe wechselte: In einem Brief an Bullinger meldet er, dass er in der Beilage auch Briefe aus Italien für Gessner habe. ${ }^{17}$ Des weiteren lassen Bündner Korrespondenten Bullingers immer wieder Gessner grüssen, was ebenfalls auf Kontakte Gessners hinweist. ${ }^{18} \mathrm{Ge}$ legentliche Empfehlungen zeigen weitere Kontakte. Gessner erbittet beispielsweise vom Churer Arzt Hieronymus Brixius eine Empfehlung an den trefflichen Arzt Quadrio und seinen Sohn, damit sie bei Lugano seltene Pflanzen suchen können. ${ }^{19}$ In der Korrespondenz Gessners mit den Bündnern ragt diejenige mit Johannes Fabricius Montanus (1527-1566), humanistischer Universalgelehrter und seit dem Tod Comanders († 1557) Pfarrer zu St. Martin in Chur, heraus. ${ }^{20}$ Diese Korrespondenz ist vor allem auch im Blick auf das „Botanisieren“ wichtig - hatte doch Fabricius regelmässig Pflanzen für Gessner gesammelt. ${ }^{21}$

Wie in anderer Humanisten- und Reformatorenkorrespondenz finden sich auch regelmässig Hinweise auf den Austausch und die Besorgung von Büchern. Gerade

16 Genannt seien: Baldassare Altieri, Albert Belfort, Iachiam Bifrun, Hieronymus Brixius, Scipione Calandrini, Johannes Fabricius Montanus, Giulielmo Gratarolo, Daniel Gugelberg v. Moos, Johannes Pontisella, Francesco Porto,

17 Vgl. Johannes Blasius an Heinrich Bullinger, 12. Dezember 1547, in: Schiess (1904-1906), Bd. 1, Nr. 92. Es mögen viele andere Briefe verloren gegangen sein - nur einige Beispiele sollen dies illustrieren: Gessner hat sich im März 1555 wegen eines verwandten Geistlichen an Pontisella gewandt, doch der Brief ist nur sekundär belegt (vgl. Schiess [1904-1906], Bd. 1, Nr. 284). Fabricius meldet am 28. August 1564 an Bullinger, dass in der Beilage noch ein Brief an Gessner sei (vgl. Schiess [1904-1906], Bd. 2, Nr. 631) - von Mitte bzw. Ende August ist aber kein Brief von Fabricius an Gessner erhalten (vgl. Leu [2016], S. 397). Dasselbe lässt sich von dem Brief sagen, in dem Ende Oktober 1564 Gessner über Bullingers Genesung berichtete (vgl. Schiess [1904-1906], Bd.2, Nr. 643). Schliesslich wissen wir, dass Gessner mit Chiampell und anderen Engadinern in den 60er Jahren im Briefwechsel stand. Chiampell schreibt in den 70er Jahren: ,..., a domino Conr. Gesnero in quadam ad nos olim epistola ... appellatur ...“ (Chiampell [1900], S. 19), oder: „Cuius cum mentionem aliquam literis ad dominum Gesnerum iniecissem, respondit sibi nihil de illa constare, ...“ (Chiampell [1900], S. 20).

18 Erwähnt seien Baldassare Altieri, Pier Paolo Vergerio, Giovanni Beccaria, Celso Martinengo u.s.w.

19 Vgl. Conrad Gessner an Johannes Fabricius, 22. September 1559, Ms E II 340, f. 233r-v (Zentralbibliothek Zürich).

20 Vgl. Leu (2016), S. 396 f.

21 Dies zeigt sich nicht nur in der Korrespondenz zwischen Gessner und Fabricius, sondern auch in derjenigen von Fabricius mit Bullinger (vgl. Schiess [1904-1906], Bd. 2, Nr. 418. 474. 539). 
die Korrespondenz Bullingers mit den Bündnern ${ }^{22}$ liefert wertvolle Angaben, wie und welche Bücher nach Zürich kamen oder von Zürich verbreitet wurden. So bittet Bullinger im Namen Gessners am 5. November 1557 Friedrich v. Salis (1512-1570), ${ }^{23}$ dessen Sohn Johannes von Januar 1557 bis Dezember 1558 in Zürich studierte, ${ }^{24}$ die Schrift über das Wormser Bad zu senden. ${ }^{25}$ Durch Friedrichs Sohn hat Gessner offenbar von derselben gehört. Nachdem sich Gessner deswegen auch an Fabricius gewandt hat, ${ }^{26}$ verspricht Friedrich v. Salis diese mitzunehmen, wenn er nach Zürich komme. ${ }^{27}$ Gessner hat im selben Brief an Fabricius die Apologia pro Christianis (Genf 1557) von Athenagoras gesandt. ${ }^{28}$ Eine weitere Schrift, die auch von Gessner besorgt wurde, belegt den Wissenstransfer in anderer Weise: Iachiam Bifrun (15061572) hat 1556 eine Beitrag („epistola“) über die Engadiner Käseherstellung verfasst und an Gessner gesandt, der eine solche Darstellung gewünscht hatte. ${ }^{29}$ Diese ,epistola“ liess Gessner Jahre später in Jodocus Willichs Ars magirica (Zürich 1563), einem diätischen Kochbuch, als Anhang drucken. ${ }^{30}$ Mit Bifrun hatte Gessner bereits im Zusammenhang mit seinen bibliographischen und sprachwissenschaftlichen Studien Austausch gepflegt: Seine Ausführungen zur rätoromanischen Sprache im Mithridates (Zürich 1555) ${ }^{31}$ belegen Gessners Kenntnis über den ersten rätoromanischen Druck, Bifruns Katechismus Fuorma da intraguidar la giuuentüna (Poschiavo 1552). ${ }^{32}$ Verschiedene Gratulations- und Empfehlungsgedichte in Druckschriften bezeugen ebenfalls den Wissenstransfer zwischen Gessner und den Drei Bünden. Negris Ovid-Ausgabe für den Schulgebrauch, gedruckt 1542 in Zürich, illustriert dies eindrücklich - hat doch Gessner dazu ein griechisches Gedicht verfasst, in dem er

22 Vgl. Schiess (1904-1906), Bd. 1-3.

23 Vgl. Pfister (2012), S. 113.

24 Vgl. Bernhard (2015), S. $113 \mathrm{ff}$.

25 Vgl. Schiess (1904-1906), Bd. 2, Nr. 40, 41. 43. Es handelt sich wohl um Paul P. Paravicinis De Masiniensium et Burmiensium thermarum ... situ, natura et miraculis ... (Mailand 1545); vgl. Leu (2016), S. 431; Bonorand (1967), S. 485; Brügger (1863), S. 36.

26 Vgl. Conrad Gessner an Johannes Fabricius, 3. Dezember 1557, Ms C 50a 25/2 (Zentralbibliothek Zürich).

27 Vgl. Friedrich v. Salis an Heinrich Bullinger, 15. Dezember 1557, in: Schiess (1904-1906), Bd. 2, Nr. 47.

28 Fabricius Kontakte zu Gessner reichen freilich in die Zeit vor seinem Churer Amtsantritt zurück: Fabricius hat Gessner Anfang der 40er Jahre kennengelernt, als er zum zweiten Mal zu Studien in Zürich weilte (vgl. Fischer [1940], S. 322-336); vide infra.

29 Es darf davon ausgegangen werden, dass Gessner den Engadiner Käse bereits gekostet hatte: Bekanntlich hat Johannes Travers solchen 1545 an Bullinger gesandt (vgl. Johannes Travers an Heinrich Bullinger, 10. September 1545, in: Schiess [1904-1906], Bd. 1, Nr. 60).

30 Vgl. Willich (1563), S. 220-227; Leu (2016), S. 150. 334; Gaudenz (1993), S. 445-451; Bonorand (1987), S. 74.

31 Vgl. Gessner (1555a), f. 65r-v; Simmler (1555a), f. 53v; ders. (1555b), f. 82v; vgl. Bernhard (2008), S. 197.

32 Vgl. Bernhard (2008), S. 187-247. 
das Büchlein zum fleissigen Studium empfiehlt. ${ }^{33}$ Dass Negri und Gessner sich auch über Druckschriften ausgetauscht haben, illustriert ein Hinweis in der Bibliotheca universalis (1545): Gessner schreibt, dass Negri im Jahr 1544 Machiavellis Discorsi sulla prima decade di Livio „ex Italicis Latinos faceret“; 34 die Schrift kam allerdings nie in den Druck. Für eine Schrift, die Fabricius „mutato nomine ad cardinalem primarium Galliae“ geschrieben hat, verfasste Gessner gleichfalls griechische Gedichte - auch diese scheinen nie erschienen zu sein. ${ }^{35}$

Es ist bekannt, wie vielschichtig im 16. Jahrhundert die Verbreitung von Druckschriften von statten ging, auch in den Drei Bünden. Es handelte sich keineswegs nur um reformatorische Schriften, sondern um Druckerzeugnisse verschiedenster Art. ${ }^{36}$ So hat sich ein weiter nicht bekannter Johannes Bartholomeus Bifrun (1535?) - er ist nicht verwandt mit dem Juristen Iachiam Bifrun ${ }^{37}$ - Senecas Tragoediae (Leiden 1548) angeschafft, ${ }^{38}$ oder Jakob Travers hat 1526 Erasmus Collectanea adagiorum veterum ... (Strassburg 1520) gekauft. ${ }^{39}$ Es ist naheliegend, dass auch Gesneriana in den Drei Bünden nicht nur gefragt waren, sondern auch bewusst verbreitet wurden. Das Vorhandensein solcher allein belegt freilich noch nicht, dass diese im 16. Jahrhundert in die Drei Bünde gekommen sind, ausser sie haben einen Besitzeintrag aus dieser Zeit. Dank bibliotheksgeschichtlichen Untersuchungen der letzten Jahre lässt sich heute immerhin sagen, dass seit den 60er Jahren des 16. Jahrhunderts unter anderem folgende Werke Gessners von Bündnern angeschafft worden sind: das Onomasticon proprium nominum (Zürich 1549), ${ }^{40}$ die Historia animalium (Zürich 1551), ${ }^{41}$ der Mithridates (Zürich 1555), ${ }^{42}$ das Kräuterbuch De raris ed admirandis Herbis (Zürich 1555) ${ }^{43}$ sowie das Lexicon Graeco-Latinum (Zürich 1556). ${ }^{44}$

Buchgeschichtlich betrachtet haben verschiedene Kontakte Gessners mit den Bündnern noch einen anderen Hintergrund: Bücher und Druckschriften kamen nicht nur von Zürich in die Drei Bünde oder wurden von hier - es ist dabei an die Druckerei Landolfi in Poschiavo zu denken ${ }^{45}$ - nach Zürich verbreitet, sondern das

33 Vgl. Conradi Gesneri Tigurini ad studiosos pueros ..., in: Negri (1542), S. 23 (= f. C 7 r); vgl. Bernhard (2010), S. $83 \mathrm{f}$.

34 Gessner (1545), f. 253v.

35 Vgl. Johannes Fabricius an Heinrich Bullinger, 6. Juni 1562, in: Schiess (1904-1906), Bd. 2, Nr. 452.

36 Vgl. Bernhard (2018).

37 Vgl. Kaiser (1954), S. 179.

38 Signatur: O 3590 (Kantonsbibliothek Graubünden).

39 Signatur: O 2992 (Kantonsbibliothek Graubünden).

40 Keine Signatur (Bibliothek Casa Battista Soglio).

41 Signaturen: F 835; F 836 (Kantonsbibliothek Graubünden).

42 Signaturen: O 3349 (Kantonsbibliothek Graubünden); 196 (Bibliothek Pult Sent).

43 Keine Signatur (Bibliothek Stamparia Strada).

44 Signaturen: 03100 (Kantonsbibliothek Graubünden); Dep. 10, 3d [Schachtel 12] (Archiv culturel Engadin'Ota).

45 In Poschiavo sind in der betreffenden Zeit über zwanzig Drucke erschienen (vgl. Bornatico [1976], S. 39-55; Bonorand [1960], S. 228-244). 
Gebiet der Drei Bünde nahm bereits seit den 30er Jahren des 16. Jahrhunderts eine zunehmend wichtigere Rolle für den Transport von Druckschriften von und nach Italien ein. Gerade in der Humanisten- und Reformatorenkorrespondenz lesen wir immer wieder von Buchlieferungen über die Bündnerpässe. So berichtet z. B. Vergerio um 1553 aus Chiavenna, dass das Fass mit Büchern aus Basel noch nicht angekommen sei, oder Comander meldet 1533 an Vadian, dass er Bücher Zwinglis, Oekolampads oder Bucers nach Italien sende, um die dortige reformatorische Bewegung zu unterstützen. ${ }^{46}$ Gerade Vadian hat viel zu den Kontakten Gessners mit Bünden, den Untertanenlanden und Italien beigetragen. So geht auch der Kontakt zum Venezianer Handschriftensammler Arnold Arlenius auf Vadians Bemühungen zurück. ${ }^{47}$ Bereits als Gessner im Sommer 1543 seine Italienreise antrat, wählte er den Weg über die Drei Bünde, wie er selbst schildert: „Cum per alpes ... in Italiam transirem, medio fere itinere inter Curiam \& Clauennam, si bene memini, in monte Speluca thermas esse audivi natura calentes.“ 48 Das war seine erste Bündnerdurchquerung über den Splügenpass nach Chiavenna und von dort, wie die Studien von Bonorand zeigen, nach Venedig zu Arlenius. Auf dem Rückweg reiste er ins Vinschgau, über den Reschen nach Feldkirch und zurück in die Schweiz. ${ }^{49}$

Der dritte wichtige Aspekt für den Wissenstransfer ist die Gelehrtenperegrination, die für die Drei Bünde nicht nur die grundlegende Form der Wissensaneignung darstellte, sondern auch eine wichtige Voraussetzung für Wissensverbreitung war. Bereits während seiner Studienzeit in Zürich hat der Bergeller Johannes Pontisella (1510-1574) Gessner kennengelernt. ${ }^{50}$ Als Gessner in Lyon im Januar 1541 an einer Buchmesse teilnahm, lernte er einen anderen Bündner kennen, den gelehrten Arzt Albert Belfort von Chiavenna, dessen Gastfreundschaft er in Lyon einige Tage geniessen durfte. ${ }^{51}$ Nach dem Doktorat in Basel kehrte Gessner nach Zürich zurück, wo die Schola Tigurina von zahlreichen Bündnern aufgesucht wurde. In den Vorlesungen über Naturphilosophie und Ethik lernten diese Gessner kennen und schätzen. Bis zu seinem Ableben haben mindestens 30 Bündner bei Gessner an Vorlesungen teilgenommen. Eine Sichtung der Namen illustriert die Personenkontakte Gessners in erweiterter Weise: Die Söhne des Staatsmanns Johann Travers, Humanist Francesco Negri, Pfarrer Johannes Blasius, Jurist Iachiam Bifrun, Arzt Nicolò Guicciardi, Apotheker Ludovico Quadrio, Podestà Johann Anton v. Planta und zwei Neffen des Reformators Pier Paolo Vergerio haben in Zürich studiert; dazu gesellen

\footnotetext{
46 Vgl. Pier Paolo Vergerio an Heinrich Bullinger, 26. Februar 1553, in: Schiess (1904-1906), Bd. 1, Nr. 205; Johannes Comander an Joachim Vadian, 10. April 1533, in: Arbenz (1890-1913), Bd. 5, Nr. 732; vgl. dazu Bonorand (2000), S. 111-120.

47 Vgl. Bonorand (1987), S. 73; ders. (1991), S. 63.

48 Gessner (1553), f. 295v. Bereits Bonorand hat dies mit guten Gründen vermutet; Leu hat sich dazu nicht geäussert (vgl. Bonorand [1987], S. 73).

49 Vgl. Leu (2016), S. 127. 416; Bonorand (1991), S. 61-63.

50 Vgl. Bonorand (1987), S. 72; vide infra.

51 Vgl. Gessner (1542), f. a4v; ders. (1545), f. 17v; Leu (2016), S. 70.
} 
sich andere Namen - teils aus berühmten Geschlechtern - wie Antonius Stuppa, Caspar Alexius, Johann Travers v. Salis, Wolfgang v. Juvalta, Matteo della Torre oder Jacob Schucan. ${ }^{52}$ Andere Bündner Studenten, die anderswo studiert haben, besuchten auf ihrer Heimreise in Zürich noch Gessner. ${ }^{53}$ Dass solche Begegnungen für Gessners Kontakte mit den Drei Bünden von grosser Bedeutung sein konnten, zeigt sich in den Quellen immer wieder. Exemplarisch sei auf den Engadiner Notar und Richter Jacob Schucan (1536-1594) verwiesen: Nach seinen Studien in Basel und Heidelberg (1551/52) hielt er sich vorübergehend im Engadin auf, um schliesslich 1557 in Zürich seine Studien noch zu vollenden, offenbar auch bei Gessner. ${ }^{54}$ Wenig später hat er Gessners Lexicon Graeco-latinum (Basel 1556) angeschaffen. ${ }^{55}$ Offenbar blieb er aber mit Gessner in Kontakt, so dass er sich bei einem weiteren Aufenthalt in Zürich im April 1565 in Gessners Stammbuch mit einem Zitat aus Vergils Aeneis verewigte: „Facilis descensus Averni.“56 Im Dezember desselben Jahres ist Gessner schliesslich an der Pest verstorben.

\section{Themata}

Die Zusammenstellung der Personen aus den Drei Bünden, mit denen Gessner sich getroffen hat bzw. mit denen er in Kontakt stand, illlustriert, dass die überwältigende Mehrheit einem reformatorischen Bekenntnis angehörten bzw. eine reformhumanistische Haltung vertrat. Aber nur etwa ein Drittel von Gessners Kontakten sind Personen, die sich hauptsächlich oder gar ausschliesslich um die Konsolidierung des reformatorischen Bekenntnisses in den Drei Bünden bemüht haben. Bekenntnisfragen sind bei den Kontakten Gessners mit Bündnern grundsätzlich sekundär. Dies sticht vor allem bei den Personen ins Auge, deren Verdienst um die Reformation bekannt ist. Pier Paolo Vergerio sendet beispielsweise ein Rezept gegen Nierensteine an Gessner - „Aqua probata ad calculum renum ...“57 Der Austausch ist insbesondere von „naturwissenschaftlichen“ Themata wie Bädern, Mineralquellen, seltenen Pflanzen, Käseherstellung oder medizinischen Fragen beherrscht. Immer wieder wird auch Gessner um Rat gefragt: Als der Churer Arzt Zacharias Beeli einer

52 Vgl. Bonorand (1949), S. 101-103.

53 So z. B. Stephan Heim, der 1562 in Heidelberg und 1563 in Basel studierte; er trug sich in Zürich am 16. Juni 1564 ins Stammbuch von Gessner ein (vgl. Conrad Gessner, Liber amicorum [Kopie], 1555-1565, Ms Z VIII 759, Nr. 205 [Zentralbibliothek Zürich]). Heim wurde der Nachfolger von Tobias Egli in Davos, starb aber bereits 1565 (vgl. Bonorand [1987], S. 77).

54 Vgl. Bonorand (1949), S. 102; ders. (1987), S. 75 f. Zu Jacob Schucan vgl. auch: Genealogia Schucanorum, B 197 (Staatsarchiv Graubünden); Truog (1938), S. 345-348.

55 Dieses Werk ist in der Kantonsbibliothek Graubünden (Chur) erhalten und hat folgenden Besitzeintrag: „Ex libris Jacobi Schucani Rhaeti“ (Signatur: O 3100).

56 Conrad Gessner, Liber amicorum [Kopie], 1555-1565, Ms Z VIII 759, Nr. 215 (Zentralbibliothek Zürich); vgl. Vergil, Aeneis VI, 126.

57 Vgl. Conrad Gessner, Thesaurus medicinae practicae ..., Ms S 204c, S. 15 (Zentralbibliothek Zürich). 
Verwandten von Stadtschreiber Daniel Gugelberg von Moos gegen ihre starken Kopfschmerzen nicht helfen konnte, wandte sich der Stadtschreiber selbst an Gessner. ${ }^{58}$ Aufs Ganze betrachtet lässt sich auch erkennen, dass mehrere Bündner Persönlichkeiten im Austausch mit Gessner zu eigenen Untersuchungen an Pflanzen, Medikamenten, Bädern usw., quasi zum „Botanisieren“, angeregt worden sind. Es sei auf den Juristen Iachiam Bifrun, den Lateinlehrer Johannes Pontisella, Antistes Johannes Fabricius, Arzt Nicolò Guicciardi, Graecist Francesco Porto oder den $\mathrm{Hu}$ manisten und Reformator Durich Chiampell verwiesen. Ein Blick in Gessners zwei Bände der Historia plantarum, deren Originale in der Universitätsbibliothek Erlangen lagern, ${ }^{59}$ offenbart zudem, wie viele Bündner Gelehrte Kräuter und seltene Pflanzen an Gessner gesandt und damit die Historia plantarum beliefert haben: Tobias Egli aus Davos sandte beispielsweise die Viola lutea, ${ }^{60}$ Zacharias Beeli das edle Mondkraut (Lunaria nobilis), ${ }^{61}$ Pontisella fand am Churer Rhein Zwergrohrkolben (Typha minor) ${ }^{62}$ oder Fabricius am Calanda Chrysanthemen. ${ }^{63}$ Von letzteren beiden wissen wir zudem, dass sie mit Dr. Zacharias Beeli im Juni 1559 auf dem Piz Calanda (bei Chur) neunzehn Pflanzen gefunden und an Gessner nach Zürich gesandt haben. ${ }^{64}$ Als Folge davon sandte Gessner einen Boten nach Chur, der mit Fabricius weitere Pflanzen hätte suchen sollen; doch als er Anfang Oktober in Chur eintraf, konnte man nicht mehr auf den Calanda - er war bereits schneebedeckt. ${ }^{65}$ Noch Anfang September 1563 berichtet Fabricius, dass er mit Pontisella den ganzen Tag in den Bergen verbracht habe, um für Gessner seltenere Pflanzen zu suchen. ${ }^{66}$

Ein anderes Beispiel betrifft zwar auch das „Botanisieren“, doch greift die „Wirkung“ Gessners noch breiter: Chiampell, den Gessner auf seiner „Bäderreise“ im Juli 1561 in Susch besucht haben muss, ${ }^{67}$ hat im Auftrag Gessners bald Pflanzen,

58 Vgl. Daniel Gugelberg von Moos an Conrad Gessner, 12. Mai 1562, Ms S 204a, f. 51v (Zentralbibliothek Zürich); vgl. Leu (2016), S. 342.

59 Vgl. Conrad Gessner, Historia plantarum, H62/MS 2386 (Universitätsbibliothek Erlangen); Conrad Gessner, Historia plantarum (Kopie), Ms Z VIII 394, 394a, 395 und 395a (Zentralbibliothek Zürich).

60 Vgl. Conrad Gessner, Historia plantarum, Ms Z VIII 394a/203a (Zentralbibliothek Zürich).

61 Vgl. Cordus (1561), f. 235v.

62 Vgl. Conrad Gessner, Historia plantarum, Ms Z VIII 395/424 (Zentralbibliothek Zürich).

63 Vgl. Conrad Gessner, Historia plantarum, Ms Z VIII 395/14 (Zentralbibliothek Zürich).

64 Vgl. Johannes Fabricius an Conrad Gessner, 26. Juni 1559, in: Cordus (1561), f. 235v; Leu (2016), S. 270.

65 Vgl. Johannes Fabricius an Heinrich Bullinger, 9. Oktober 1559, in: Schiess (1904-1906), Bd. 2, Nr. 201.

66 Vgl. Johannes Fabricius an Heinrich Bullinger, 6. September 1563, in: Schiess (1904-1906), Bd. 2, Nr. 539.

67 Ich gehe, nach Auswertung aller Quellen zu Gessner und Chiampell, über die bisherige Ansicht hinaus (vgl. Leu [2016], S. 327; Steiger [1978], S. 220; Bonorand [1987], S. 77; Brügger [1863], S. 40f). Zudem müssen Chiampells Formulierungen der Darstellung von Gessners Besuch von Vulpera auf einer persönliche Erinnerung basieren (vgl. Chiampell [1884], S. 210). Eine kritische Edition von Chiampels Raetias alpestris topographica descriptio erscheint 2019, herausgegeben vom Institut für Kulturforschung Graubünden. 
Proben und gar eine ausführliche Beschreibung einer merkwürdigen eiskalten periodischen Quelle in der Val d'Assa (Unterengadin) übersandt. ${ }^{68}$ Doch blieb es nicht dabei: Als Humanist hat sich Chiampell fortan intensivst mit den Schriften Gessners auseinandergesetzt. Diesbezügliche Einflüsse zeigen sich im äusserst interessanten Anhang (Cap. 50-57) zur Raetiae alpestris topographica descriptio, die allerdings erst um 1900 im Druck erschien. ${ }^{69}$ Darin werden unter anderem Naturmerkwürdigkeiten, Mineralprodukte, Tiere, Pflanzen, Bäder und anderes behandelt. Es erstaunt daher wenig, dass Chiampell seinen Ausführungen neben Stumpf namentlich Werke von Gessner zugrundlegt - sei dies bei der Beschreibung von Kräutern und Wurzeln (z. B. Baldrian) oder der Darstellung von Tieren (z. B. Geier). Die von ihm benutzten Quellen sind insbesondere die Icones animalium quadrupedum ... (Zürich $1555)^{70}$ sowie seine berühmte Abhandlung Horti Germaniae, die in Valerius Cordus' Annotationes in Pedacii ... (Strassburg 1561) als Anhang gedruckt worden ist. ${ }^{71}$

\section{Provenienz der Personen}

Ein Blick auf die Herkunft der Personen, mit denen Gessner länger Kontakt pflegte, zeigt eine Konzentration auf das Gebiet um Chur, das Oberengadin (Samedan), das Bergell, die Valchiavenna sowie das Veltlin. Kontakte aus andern Gebieten sind vorübergehend oder zufällig. Weit mehr als die Hälfte der Kontakte betraf den Austausch mit Bewohnern der bündnerischen Südtäler bzw. italienischen Glaubensflüchtlingen, darunter auch mehrere Ärzte und Literaten, die in ihrem Bekenntnis „humanistische Offenheit“ signalisierten. Man denke an Guglielmo Gratarolo, Francesco Porto, Nicolò Guicciardi oder Nicolò Pestalozzi. ${ }^{72}$ Was die Personenkontakte

68 Die Beschreibung der Quelle in der Val d'Assa ist gedruckt in: Chiampell (1884), S. 217-219. Am 23. Oktober 1562 sprach Gessner seinen Dank an Chiampell für die übersandten Informationen aus (vgl. Conrad Gessner an Johannes Fabricius, 23. Oktober 1562, Ms C 50a, Nr. 41/2 [Zentralbibliothek Zürich]); Caduff (2012), S. 108-113; Bonorand (1987), S. 77; Schiess (1899-1900), S. 5f; Brügger (1863), S. $41 \mathrm{f}$. Dieser merkwürdige Bach scheint in Zürich eine solche Verwunderung erregt $\mathrm{zu}$ haben, dass er noch in einem Schreiben an Pontisella erwähnt wird (vgl. [Ludwig Lavater?] an Johannes Pontisella, s.d., in: à Porta [1771], Bd. I/1, f. b ${ }_{2} r-c_{2} r$ [= Schiess (1904-1906), Bd. 3, Nr. 457]). Dieser Brief wird in der Geschichtsschreibung seit der Edition von à Porta - das Original ist heute verloren - Bullinger zugeschrieben, kann aber nach heutiger Erkenntnis nicht von Bullinger stammen. In demselben wird nämlich ein Besuch des Briefschreibers in den Drei Bünden erwähnt (,vidi maximum in arce Haldenstein“), was jedenfalls für Bullinger nicht zutrifft. Mehrere Indizien sprechen dafür, dass der Brief Ende der 70er-Jahre von Ludwig Lavater verfasst wurde (freundliche Mitteilung von lic.theol. Rainer Henrich, Winterthur).

69 Vgl. Chiampell (1900), S. 1-121.

70 Wie Schiess nachweisen konnte, muss es sich um die 1. Auflage handeln (vgl. Schiess [19001901], S. CIV).

71 Vgl. Gessner (1561), f. 236r-300r.

72 Nicolò Pestalozzi stammte von Chiavenna und war als Notar, später als Stadtrat in Chiavenna tätig; er pflegte regelmässig Kontakt zur Zürcher Familie Pestalozzi. Die Quellen zu N. Pestalozzi 
betrifft, ist erwähnenswert, dass die italienischen Kontakte auch mehrere Personen aus Romanischbünden vermittelten.

\section{Gessners Interesse an den Drei Bünden}

Nach den bisherigen Ausführungen liegt es nahezu auf der Hand, dass Gessners Hauptinteresse an den Drei Bünden bei „Kräuter“-Fragen lag. Eine Untersuchung der Anfänge und Formierung der Kontakte Gessners erlaubt aber Differenzierungen. Mit Recht betont Bonorand, dass die Anfänge der Beziehungen Gessners zu den Drei Bünden in die Jugendzeit zurückreichen, als Gessner in Zürich Lehrer an der Lateinschule (1535-1537) war und Johannes Pontisella, Sohn eines Bergeller Domherrn, seine Studien in Zürich absolvierte. ${ }^{73}$ Bis an sein Lebensende war Gessner mit Pontisella in Freundschaft verbunden. Nach einem kürzeren Engagement als Pfarrer von Schwamendingen und Provisor am Carolinum wurde Pontisella 1544 an die städtische St. Nikolaischule in Chur berufen, wo er bis zu seinem Tode wirkte. ${ }^{74}$ Aus den Jahren nach 1544 bis zum Amtsantritt von Fabricius Montanus in Chur (1557) sind allerdings kaum Quellen erhalten, die Hinweise zum Austausch Gessners mit Pontisella geben könnten. Die in dieser Zeit belegten Kontakte betreffen eher Personen aus Chiavenna - die Kontakte in Chiavenna gehen auf die Italienreise 1543 zurück - und später aus dem Oberengadin. Das „Botanisieren“ ist in diesen Quellen aber kaum Thema. Vielmehr beschäftigten Gessner andere Themata, beispielsweise bio-bibliographische oder sprachwissenschaftliche Fragen. Es scheint, dass Gessner das erste Mal mit der rätoromanischen Sprache in den frühen 50er Jahren in direkten Kontakt kam. Als in Poschiavo 1552 der erste rätoromanische Druck, der Katechismus Vna cuorta et christiauna fuorma da intraguider la giuuentü$n a$, gedruckt worden ist, scheint Gessner sich für diese - wie er schreibt - zunehmend ,verdorbene“ Sprache (corruptissima lingua) ${ }^{75}$ stärker interessiert zu haben und den einen oder anderen romanischsprechenden Studenten in Zürich nach dem Text des Herrengebetes gefragt. ${ }^{76}$ Bekannt ist zumindest, dass im November 1554 Wolfgang v. Juvalta († 1586) aus Zuoz, nachdem er an der Nicolaischule in Chur bei Pontisella unterrichtet worden ist, mit einer Empfehlung von Johannes Travers nach

sind sehr verstreut und schwer greifbar (vgl. unter anderem: Bonorand [1987], S. 78; PestalozziKeyser, Hans: Histoire de la famille Pestalozzi: http://protestantsdanslaville.org/gilles-castelnaugenealogie/Pestalozzi.htm).

73 Vgl. Bonorand (1987), S. 72.

74 Vgl. Bernhard (2016), S. 347; Gillardon (1907), S. 14; Schiess (1904-1906), Bd. 1, S. XLIIIff; ders. (1903), S. $21 \mathrm{ff}$.

75 Vgl. Gessner (1555), f. 65r-v; Simmler (1555a), f. 53v; ders. (1555b), f. 82v.

76 Bifruns Verdienst erwähnt Gessner hingegen lobend in seiner Epistola nuncupatoria zu Willichs Ars Magirica (vgl. Willich [1563], f. *6v-7r). 
Zürich kam. ${ }^{77}$ Juvalta, später Landammann des Oberengadins und Gesandter in Venedig, ${ }^{78}$ stand im Austausch mit Friedrich v. Salis; ${ }^{79}$ zudem war er, wie eine Marginalie Gessners zum Eintrag seines Bruders Johannes ins Stammbuch belegt, „conjunctus aut affinis d. Jac. Bifrontis." 80 Dass das Herrengebet, wie es Gessner im Mithridates (1555) abgedruckt hat, nicht identisch ist mit demjenigen, das Bifrun in seiner Fuorma (1552) publiziert hat, ${ }^{81}$ belegt immerhin, dass Gessner den Katechismus nicht zur Hand hatte, er für das Herrengebet also eine andere „Quelle“ benutzt haben musste. ${ }^{82}$ Vieles spricht dafür, dass Wolfgang v. Juvalta Gessners Quelle war - bekanntlich war seine Familie mit den Familien v. Salis-Samedan, Travers und Bifrun verschwägert. Des letzteren Sohn hat ums Jahr 1555 auch in Zürich Studien absolviert. ${ }^{83}$ Eine Folge dieses Austausches mit Bündner Studenten war, dass Gessner mit Iachiam Bifrun und Friedrich v. Salis in Kontakt trat. Wie erwähnt, wurde Bifrun von Gessner gebeten, über die Käseherstellung im Engadin zu berichten, v. Salis sollte Gessner die Schrift über das Wormser Bad überlassen. ${ }^{84}$ Im Januar 1557 hielt sich v. Salis schliesslich selbst in Zürich auf. ${ }^{85}$ Später sollten sowohl der Jurist und Linguist Bifrun als auch der Politiker und Reformationsfreund v. Salis Gessner bei seinen botanischen und balneologischen Studien behilflich ein. ${ }^{86}$ Die Korrespondenz Gessners mit Fabricius ist eine wertvolle Quelle für detailliertere Angaben zu Gessners Interesse an den Drei Bünden und dessen Kontakten mit den dortigen Gelehrten.

77 Vgl. Schiess (1904-1906), Bd. 1, Nr. 273; Bonorand (1949), S. 102.

78 Vgl. Bonjour, Edgar: Fortunat v. Juvalta, in: HLS 7, S. 31; Ardüser (1598), S. 61-63.

79 Vgl. Briefe und Berichte der Familien Travers und Salis-Samedan, Ms B 1804 (Staatsarchiv Graubünden): Johannes Travers an seinen Vetter Johannes Travers von Salis, 24. Februar 1559, Ms A Sp III/8y IV.02 (Staatsarchiv Graubünden).

80 Johannes v. Juvalta hatte einst mit Schucan in Basel und Heidelberg studiert (vgl. Bonorand [1987], S. 77f); gegen Ende April kam er nach Zürich und übergab Bullinger einen Brief von Fabricius (vgl. Schiess [1904-1906], Bd. 2, Nr. 689) und trug sich am 28. April ins Stammbuch von Gessner ein (vgl. Conrad Gessner, Liber amicorum [Kopie], 1555-1565, Ms Z VIII 759, Nr. 215 [Zentralbibliothek Zürich]).

81 Vgl. Bifrun [1552], S. 17; Gessner [1555], f. 65r. Die Unterschiede sind auf die regional verschiedene Aussprache zurückzuführen.

82 Es seien einige Beispiele genannt: Bifrun schreibt tschil (Himmel), Gessner cschil, Bifrun l'g tieu num (dein Name), Gessner ilg tes num, Bifrun schreibt nuns mnêr (führe uns nicht), Gessner nun ens mener, Bifrun schreibt dalg mêl (vom Bösen) und Gessner da tuots mels (von allem Bösen) (vgl. Bernhard [2008], S. 233). Gessners Version wurde schliesslich in ganz Europa verbreitet und hat in verschiedenen „polyglotten“ Werken Niederschlag gefunden, so z. B. in Bocatius’ Oratio Dominica polyglottos (Kaschau 1614); vgl. Bernhard (2014), S. $7 \mathrm{f}$.

83 Vgl. Pietro Parisotto an Heinrich Bullinger, 8. Mai 1556, in: Schiess (1904-1906), Bd. 1, Nr. 313; Iachiam Bifrun an Conrad Gessner, 27. Januar 1556, in: Willich (1563), S. 220.

84 Vide supra.

85 Vgl. Bernhard (2015), S. 114.

86 Vgl. Conrad Gessner an Johannes Fabricius, 19. Juli 1560, in: Gessner (1577), f. 88r-v; Conrad Gessner an Johannes Fabricius, 3. September 1561, in: Gessner (1577), f. 90v-91r. 
Mit dem Amtsantritt von Fabricius Montanus an der Churer Martinskirche (1557) rückte Chur und seine Umgebung stärker in den Blick von Gessner. Ja, man kann gar sagen, dass Gessners Interesse an den Drei Bünden seit 1557 grundsätzlich grösser wurde - stand Gessner mit Fabricius doch bereits seit 1551, als Fabricius in Zürich die Leitung der Fraumünsterschule übernommen hatte, in regelmässigem Austausch. ${ }^{87}$ Der neu auflebende freundschaftliche Kontakt mit Pontisella zeigte sich unter anderem darin, dass Pontisella begann für Gessner in Chur und der Umgebung zu botanisieren - und zwar vor allem zusammen mit Fabricius. Als Gessner seine letzte Alpenreise antrat, traf er am 15./16. Juni in Chur natürlich auch seinen alten Freund Johannes Pontisella. Wie sehr Gessner diese Freundschaft schätzte, illustriert seine Epistola nuncupatoria in Willichs Ars Magirica (Zürich 1563), die Gessner Pontisella widmete. Er spricht darin von „vetus amicitia nostra“, dass Pontisella „multa hactenus beneficia ... contulit.“ Schliesslich lässt er am Ende seiner Epistola noch die Churer Geistlichen Fabricius und Gallicius sowie die Churer Ärzte Zacharias Beeli und Hieronymus Brixius grüssen. ${ }^{88}$ Die Freundschaft Gessners mit Pontisella betraf auch dessen Familie. Dies illustriert die Feststellung, dass, als Gessner am 13. Dezember 1565 jäh aus dem aktiven und wissenschaftlich sehr fruchtbaren Leben gerissen wurde, der Sohn von Johannes Pontisella, gleichfalls mit Namen Johannes (1552-1622), zu den Bündnern gehörte, die ein Trauergedicht auf den Tod von Gessner verfassten: „Hac requiescit humo Gesnerus, regnat in astris / Spiritus, in terris gloria summa viget. ... Iohannes Pontisella Iunior Anno aetatis suae 14." 89

Die Freundschaft mit Pontisella illustriert das polyhistorische Interesse Gessners an den Drei Bünden: Wasser, Kräuter, Käse und eine Prise Rätoromanisch. Welchen Einfluss Gessner auf seine freundschaftlichen Kontakte hatte, zeigt sich gleichfalls eindrücklich an dem Kontakt zwischen Gessner und Fabricius. Seit seinem achtzehnten Lebensjahr war Fabricius dichterisch tätig. So veröffentlichte er 1556 in Zürich seine Poemata - Gedichte aus der Zeit von 1545 bis $1555 .{ }^{90}$ Durch die Begegnung mit Gessner widmete er sich seit 1553 aber immer mehr naturwissenschaftlichen Themata. In seinen Differentiae animalium quadrupedum locos communes (Zürich 1555) klassifizierte er, aus antiken und späteren Autoren schöpfend, die Vierfüssler anhand alphabetisch aufgeführter physiologischer Eigenheiten wie Fort-

87 Vgl. Bächtold, Hans Ulrich: Johannes Fabricius Montanus, in: HLS 4, S. 364; Döpp (1998), S. 20. 30; Fischer (1940), S. 325f; Schiess (1904-1906), Bd. 2, S. XIIIff; Fabricius schreibt davon auch in seinen lateinischen Autobiographien: Döpp (1998), S. 38 und 44; Vulpinus (1894). S. 15 und 25; Johannes Fabricius: Vita ..., in: Miscellanea (1724), S. 390 f. und 402.

88 Vgl. Conrad Gessner: Epistola nuncupatoria, in: Willich (1563), f. *6r-7r. In diesem Zusammenhang ist auf einen zwischenzeitlich in Vergessenheit geratenen griechischen Brief Gessners an Pontisella zu verweisen, den Rudolf Steiger bei seinen minutiösen Studien gefunden hat: Conrad Gessner an Johannes Pontisella, 7. August 1562, Ms C 50a, f. 283r (Zentralbibliothek Zürich).

89 Simmler (1566a), f. I4r (= f. 36r).

90 Vgl. Döpp (1998), S. 8f; Vulpinus (1894). 
pflanzung und Ernährungsweise. Im Vorwort verweist Fabricius dankend und bewundernd auf Gessner. ${ }^{91}$ Mit der Übersiedlung nach Chur hat zwar Fabricius auch einzelne theologische Studien veröffentlicht, ${ }^{92}$ doch hat er auf Wunsch Gessners in seiner Freizeit vor allem botanisiert. In seinem Pfarrgarten in Chur legte er gar ein Versuchsgärtchen für Alpenpflanzen an. ${ }^{93}$ Es erstaunt daher kaum, dass Fabricius nach 1556 nur noch gelegentlich dichterisch tätig war, und wenn, dann im Sinne einer Art Wissenschaftspoesie: Ende Juli 1561 sandte er Gessner ein Gedicht über die Heilquellen bei Scuol, darunter auch über eine Salzquelle bei Tarasp. ${ }^{94}$ Chiampell war schliesslich der erste, der in seiner Raetiae alpestris topographica descriptio dies erstmals erwähnte: „... hic [i.e. Fabricius] et versibus aliquot eruditis descripsit atque ornavit.“95 Später wurde dieses Gedicht an verschiedenen Orten abgedruckt. ${ }^{96}$ Sogar Fabricius Dichtung war also von naturwissenschaftlichen Themata beherrscht. Als Zeichen des Dankes für die Zusendung der vielen Kräuter, Blumen und Samen hat Gessner, als Fabricius im Juni 1565 zum letzten Mal in Zürich weilte, ihn zu einem „parco prandiolo“ eingeladen. ${ }^{97}$

Gessners ursprüngliches Interesse an den Drei Bünden waren vor allem die Bäder: Wohl bereits 1543 hat er auf der Durchreise nach Italien Pfäfers und Fideris besucht. ${ }^{98} \mathrm{Zu}$ Beginn der 50er Jahre hat sich Gessner erneut mit den Bädern Bündens beschäftigt, nachdem Guglielmo Gratarolo Gessner einen Bericht De Thermis Rhaeticis \& Vallis Treschurij Agri Bergomatis zusandte. ${ }^{99}$ Gratorolo hat ihm schliesslich auch eine Beschreibung des Heilbades in Le Prese (Puschlav) zukommen lassen. ${ }^{100}$ Im Herbst 1557 begehrte Gessner, nicht nur das oben genannte Buch über das Wormser Bad, sondern plante, selbst in das sogenannte Wormserbad im heuti-

91 Vgl. Johannes Fabricius Montanus: Epistola nuncupatoria, in: Fabricius (1555), f. $A_{2}$ v; vgl. Leu (2016), S. 196; Fischer (1940), S. 325.

$92 \mathrm{Zu}$ seinen Publikationen vgl. Döpp (1998), S. 7f; Vulpinus (1894), S. 3 f.

93 Vgl. Gessner (1561), f. 235v. 238r. 299r; Schiess (1904), S. 305.

94 Vgl. Johannes Fabricius an Heinrich Bullinger, 14. Juli 1561, in: Schiess (1904-1906), Bd. 2, Nr. 354. Es scheint, dass Fabricius über die Salzquelle bei Tarasp, die sogenannte Halicrene, bereits vor der Reise Gessner ein Gedicht gesandt hat (vgl. Conrad Gessner an Johannes Fabricius, 20. März 1561, in: Gessner [1577], f. 89v-90r).

95 Chiampell (1884), S. 210.

96 Vgl. Miscellanea (1724), S. 404-406; à Porta (1771), Bd. I/2, S. 337-340. Neuzeitliche Edition bei Döpp (2012), S. 39-45. Zum Ganzen vgl. Scheuchzer (1717), S. 303-305; Döpp (2012), S. 9-15.

97 „Ovis \& cerasis libetne mecum / Parco prandiolo, Fabrice amice, / Esurire parumper? Hora nona / Nostras te moneat venire in aedes.“ (Conrad Gessner an Johannes Fabricius, Juni 1565, in: Miscellanea [1724], S. 404). Fabricius hat auf den Tod von Gessner auch ein Trauergedicht verfasst (vgl. Simmler [1566], f. 35r) und beklagt gegenüber Bullinger den Druckfehler nequit statt nequiit (vgl. Johannes Fabricius an Heinrich Bullinger, 9. April 1566, in: Schiess [1904-1906], Bd. 2, Nr. 773). 98 Vgl. Gessner (1553), f. 294r-295r; Leu (2016), S. 328.

99 Vgl. Wilhelm Gratarolo an Conrad Gessner, s.d., in: Giunta (1553), f. 192r; vgl. Döpp (2012), S. $26 \mathrm{f}$.

100 Vgl. Milt (1945), S. 4. 
gen Bormio zu reisen. ${ }^{101}$ Die intensiveren Kontakte mit den Churer Gelehrten haben aber Gessners Interesse für die Drei Bünde verlagert. Seine Bäderreise von 1561 ist schliesslich, wie die Studien von Brügger, Steiger, Döpp und Leu ohne Zweifel belegen, vom Botanisieren geprägt. Er sammelte Kräuter, besuchte Gärten und interessierte sich für Heilmittel. Dies illustriert, dass Gessner nicht nur mehrere Bündner $\mathrm{zu}$ „naturwissenschaftlichen“ Tätigkeiten ermutigte, sondern als Folge der Intensivierung der Kontakte auch bei ihm selbst eine Verlagerung der Interessen stattgefunden hat. Rosius à Porta selbst schrieb über die Reise von 1561, dass Gessner vielfältige Gründe für die Reise gehabt hatte: „Conradus Gessnerus Raeticas alpes invisit, harumque beneficia, herbas, istarumque virtutes, accuratius scrutatus est ...."102

\section{Zusammenfassung und Ertrag}

Die seit den 30er Jahren bestehenden einzelnen Kontakte mit einzelnen Bündnern wurden einerseits durch Gessners Bündner-Reisen sowie andererseits durch Bullingers bedeutende Korrespondenz zunehmend ausgebaut. Gessner verfügte seit den 50er Jahren über ein breites Netzwerk in den Drei Bünden. Dazu trugen freilich Gessners intensive Kontakte mit den beiden ehemaligen Zürcher, später aber in Chur wirkenden Gelehrten Pontisella und Fabricius nicht unwesentlich bei. Sie waren es auch, die Gessners Interesse an den Drei Bünden als Forschungsfeld für die Botanik weckten. Doch nicht nur Pflanzen sandten die Bündner - erwähnt sei beispielsweise der sonst der sonst wenig bekannte Pfarrer Johannes Möhr aus Chur ${ }^{103}$ an Gessner, sondern Fabricius war gleichfalls darum besorgt, dass Gessner regelmässig einen guten Tropfen Wein erhielt. Am 11. November 1560 schrieb Fabricius an Bullinger, dass er „vina Rhetica“ für Gessner gekauft habe, ${ }^{104}$ oder im August 1565 bittet er Gessner um Geduld - er möge wegen des Weines bis zum Herbst warten. ${ }^{105}$

101 Vgl. Conrad Gessner an Johannes Fabricius, 3. Dezember 1557, Ms C 50a, Nr. 25/2 (Zentralbibliothek Zürich); vgl. Leu (2016), S. 326; Brügger (1863), S. 36.

102 à Porta (1771), Bd. I/2, S. 336.

103 Vgl. Steiger (1978), S. 221. Steiger nennt Möhr mit seinem lateinischen Namen Marius; bekannt ist er auch unter Merus (Synodalmatrikel 1557). Steiger konnte aber, wie auch Jakob Rudolf Truog, Marius nicht mit Möhr, dem späteren Pfarrer von Grüsch, identifizieren (vgl. Steiger [1978], S. 221). Möhr war in den 1560er Jahren u.a. Lehrer in Winterthur.

104 Vgl. Johannes Fabricius an Heinrich Bullinger, 11. November 1560, in: Schiess (1904-1906), Bd. 2, Nr. 291 (Beilage).

105 Vgl. Johannes Fabricius an Heinrich Bullinger, 9. August 1565, in: Schiess (1904-1906), Bd. 2, Nr. 712. 
Die Kontakte gestalteten sich vielfältig. Dazu gehört auch, dass Gessner den Churer Bürgerrat Johannes Tscharner, ${ }^{106}$ der ihn auf der Reise zum Wormserbad begleitet hatte, und den Arzt Nicolò Guicciardi aus Teglio, bei dem Gessner seinerzeit übernachtete, mit einem Schreiben an Johannes Bauhin d. J. (1541-1613), der in Lyon als Arzt wirkte, empfahl. ${ }^{107}$ Und nicht erstaunlich ist es, dass sich unter den Trauergedichten auf den Tod Gessners ${ }^{108}$ neben bekannten Namen wie Rudolf Gwalther, Théodore de Bèze, Johannes Crato von Krafftheim, Johannes Sambucus oder Nicolaus Reusner auch die Namen von Bündner Gelehrten befinden, allen voran Francesco Porto, dessen Garten Gessner seinerzeit in Chiavenna bewundert hatte. ${ }^{109}$ Sein griechisches Trauergedicht steht an erster Stelle sämtlicher Carmina doctorum virorum in obitum D. Conr. Gesneri scripta. ${ }^{110}$

Die Verbreitung von Gesneriana - wir erinnern uns daran, dass Friedrich v. Salis kurz nach Gessners Tod dessen Historia animalium begehrte ${ }^{111}$ - und der literarische Niederschlag von Gessners Kontakten mit den Drei Bünden bei den Historikern Durich Chiampell, Fortunat Sprecher von Bernegg ${ }^{112}$ oder Rosius à Porta bewahrten die Erinnerung an Gessner bis in die heutige Zeit. Die Auswertung sämtlicher Quellen wird freilich noch manches Detail und manche Präzisierung zu Gessner Kontakten mit den Drei Bünden offenbaren, denn es gilt gerade bei diesem Thema: „Nihil tam difficile quin quaerendo investigari possit. “113 Dieses Zitat von Terenz schrieb Dr. Hieronymus Brixius 1561 ins Stammbuch von Gessner. ${ }^{114}$

106 Zum Churer Bürgermeister Johannes Tscharner vgl. Brügger (1863), S. 38f; in der Korrespondenz Bullingers mit den Graubündnern wird Tscharner gelegentlich erwähnt (vgl. Schiess [19041906], Bd. 1-3).

107 Vgl. Conrad Gessner an Johannes Bauhin, 30. Juni 1564, in: Bauhin (1591), S. 145. Nebenbemerkung: Bauhin hat seinerzeit Gessner auf der Bündnerreise begleitet (vgl. Bonorand [1987], S. 75; Steiger [1978], S. 216-221).

108 Bullinger hat den Tod Gessners am folgenden Tag an Fabricius gemolden (vgl. Heinrich Bullinger an Johannes Fabricius, 14. Dezember 1565, in: Schiess [1904-1906], Bd. 2, Nr. 738).

109 Vgl. Bonorand (1987), S. 78; Steiger (1978), S. $216 \mathrm{f}$.

110 Vgl. Simmler (1566a), f. H3v (= f. 31v).

111 Vgl. Friedrich v. Salis an Heinrich Bullinger, 14. Juli 1567, in: Schiess (1904-1906), Bd. 3, Nr. 21. Da aber Gesneriana offenbar teuer waren, sah v. Salis kurz darauf davon ab, alle anzuschaffen (vgl. Friedrich v. Salis an Heinrich Bullinger, 1. September 1567, in: Schiess [1904-1906], Bd. 3, Nr. 29).

112 Vgl. Sprecher von Bernegg (1999), S. 370.

113 Terenz, Heautontimoroumenos 4, 2, 8.

114 Vgl. Conrad Gessner, Liber amicorum, 1555-1565, Ms Z VIII 759, Nr. 149 (Zentralbibliothek Zürich). 